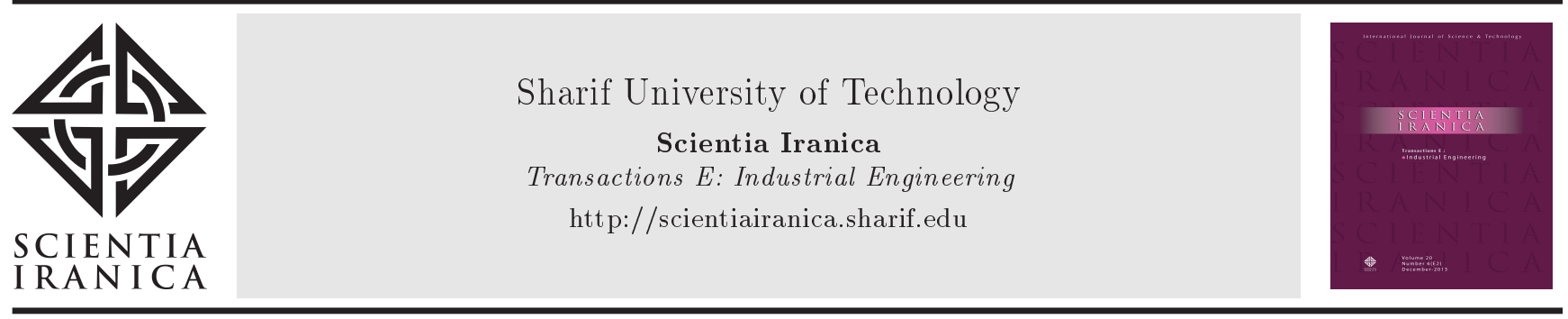

\title{
A POMDP framework to find optimal policy in sustainable maintenance
}

\author{
R. Ghandali, M.H. Abooie*, and M.S. Fallah Nezhad \\ Department of Industrial Engineering, Yazd University, Yazd, Iran.
}

Received 31 October 2017; received in revised form 1 September 2018; accepted 27 October 2018

\author{
KEYWORDS \\ Condition based \\ maintenance; \\ Sustainability; \\ Partially observable \\ Markov decision \\ process; \\ Stochastically \\ deteriorating systems; \\ Incremental pruning; \\ Accelerated vector \\ pruning; \\ Perseus.
}

\begin{abstract}
The increasing importance of maintenance and a cleaner environment besides the relations between them has encouraged the current authors to investigate a mathematical Markovian model for the condition-based maintenance problem while considering environmental effects. In this paper, the problem of proposing a maintenance optimal policy for a partially observable, stochastically deteriorating system is studied in order to maximize the average profit of the system with sustainability aspects. The modeling of this Condition-Based Sustainable Maintenance (CBSM) problem is done by mathematical methods such as Partially Observable Markov Decision Process (POMDP) and Bayesian theory. A new exact method, called accelerated vector pruning method, and other popular estimating and exact methods are applied and compared for solving the presented CBSM model, and several managerial conclusions are obtained.
\end{abstract}

(C) 2020 Sharif University of Technology. All rights reserved.

\section{Introduction}

In this section, first, the significance of maintenance and, then, its relation to environmental considerations and sustainability concept will be described.

Nowadays, the increasing importance of maintenance to all industries is undeniable $[1,2]$, and it is obvious that the magnitude of this importance varies for various systems. The priorities of all parts are not equal in one given system, and selecting a proper maintenance technique for each component has become an important topic since the latter half of the 1980s. For this reason, maintenance strategies have been developed. A maintenance strategy in a system forms

\footnotetext{
*. Corresponding author. Tel.: +98 3531232414; Fax: +983538211792

E-mail addresses: rzyghandali@gmail.com (R. Ghandali); mhabooie@yazd.ac.ir (M.H. Abooie)
}

doi: $10.24200 /$ sci.2018.5490.1306 a framework to install specific maintenance techniques for each part [3]. Reliability Centered Maintenance (RCM), Total Productive Maintenance (TPM), and Risk-based Maintenance (RBM) are the most important examples of maintenance strategies.

Some recent studies on RCM, TPM, and RBM strategies, as well as their development and implementation, can be found in [4-6].

The specific based maintenance techniques enjoy unique principles for solving maintenance problems [7]. Time-based Maintenance (TBM) and Condition-based Maintenance (CBM) are the most important maintenance techniques.

When the reliability concept emerged in 1980 , Preventive Maintenance (PM) and TBM technique were introduced, respectively. For TBM, after a specific period of operations (or time), the failure rate of products increases based on a function called the bathtub curve.

However, the deterioration rate of a system depends on not only the operation time duration but 
also various other factors and operational conditions. Therefore, the real condition of the system cannot be identified by the TBM strategy. Moreover, this strategy sometimes imposes unnecessary treatments, which often disrupt normal operations [8]. A perfect survey of such models is given in [7].

Since the TBM limitations were known in 1975 , the CBM technique was developed to maximize PM efficiency. CBM as a predictive method is the most modern and popular maintenance technique discussed in [7,9-11]. Authors [7] expressed that CBM application was more beneficial and realistic than traditional TBM approach. Therefore, CBM is usually applied to high-priority components based on their health condition.

Data acquisition is introduced as the first main step in the case of CBM [12], which requires monitoring the system's health condition. CBM estimates the health condition of equipment by real-time data received from condition-monitoring tools to make maintenance decisions and reduce unnecessary maintenance and related costs [13]

Since the application of this technique including the task of providing monitoring tools and data processing tools is not cost effective, it can be concluded that accurate and integrated planning should be considered necessary in CBM planning. Considering more than one objective and integrating them into an optimization mathematical model is more desirable and realistic from a decision-maker's point of view; therefore, a multi-objective optimization model can be useful to formulate a CBM program.

Ecological and industrial objectives, which have been considered as antagonists many times, are now taken into account simultaneously in the sustainability concept to support social, economic, and environmental pillars [14]. Balancing environmental and societal objects with financial success is no longer a matter of choice, but a must [15].

Sustainable development in general is defined as "development that meets the needs of the present without compromising the ability of future generations to meet their own needs" [16]. With planning systems characterized by low negative impact on the environment, people can participate in sustainable development effectively [17].

Recently, many researchers in various fields have turned an attentive eye to the concept of sustainability. For instance, authors on supply chain design [18] and on electric power systems $[19,20]$ attempted to include sustainability considerations into their studies.

In the maintenance concept as the main part of industry, environmental considerations can affect a problem, too. Ignoring the deterioration process of a part can lead to the failure of other related perfect parts and cause different damages to humans and the environment (such as waste and $\mathrm{CO}_{2}$ emission due to the use of old, outdated or ill-maintained equipment) besides the economic losses (such as heavy emergency replacement costs related to unexpected breakdowns). Moreover, over-maintenance by spending greater costs (more maintenance-related costs such as employment cost of maintenance workers and more part replacement costs), generating more waste (more useless replaced parts), and increasing error probability can be a risk factor in the production system and the surrounding environment. Therefore, maintenance decisions should be optimized to achieve economic, social, and environmental goals in the best feasible way.

In manufacturing businesses, sustainable maintenance can be defined as the maintenance that satisfies the objectives of a system by considering sustainability goals and constraints such as those that are related to $\mathrm{CO}_{2}$ emission, defective and non-recyclable products, useless replaced parts, scrapped items, toxic material emission (such as cleaning material utilized for repair work), and other factors that affect the environment.

Sustainability considerations are often taken into account in manufacturing processes; however, maintenance-oriented studies with sustainability considerations are still in the early stages [21].

In the rest of this section, papers in the literature related to sustainable maintenance will be divided into two main categories: conceptual and mathematical studies. Then, mathematical studies in the maintenance field will be divided into two groups: TBMrelated and CBM-related studies.

In the conceptual studies category, the following approaches were expressed in [22] that help companies improve their sustainability performance: minimizing waste production, using the best practice in maintenance, and producing process, improving the usage of metalworking fluids, lubricating oils and hydraulics oils, and so on. In [23], maintenance performance and maintenance quality and its impact on sustainability performance were studied. They concluded that maintenance processes had significant impacts on the businesses in terms of economic, environmental, and societal implications of the commercial activities. In [24], defining key performance measures for sustainable maintenance at operational and strategic levels (15 measures at the corporate level, 20 measures at the tactical level, and 43 measures at the functional level) was proposed. In [25], a sustainability-based framework for the maintenance strategy selection problem was studied. A fuzzy method was used to select the most appropriate maintenance strategy. However, the optimization model for one specified maintenance technique was not proposed.

Moreover, in a recent study [26], a comprehensive definition of sustainable maintenance was proposed in which several simple and complicated aspects such as 
economics, society, and environment besides manufacturing, overhauling, assembly, and ecology should be considered when maintenance actions were performed.

Conceptual studies mentioned above generally do not provide any mathematical programming or optimization modeling; however, basic concepts have been expressed in detail. In the following, the mathematical studies will be outlined in two groups: TBM-related and CBM-related studies.

Studies in the TBM-related group include sustainable maintenance modeling considering degradation process as a function of the system's lifetime, production rates, or so on. Some of these studies done in recent years will be introduced in the following.

In [27], a production-maintenance mathematical model for a deteriorating environmental-friendly manufacturing system was proposed that invests in $R \& D$ to make this productive technology more sustainable and avoid government-related taxation. Production rate, maintenance rate, and pollution $R \& D$ investment rate as decision variables were determined in the aforementioned model to minimize the total cost including inventory costs, production costs, emission tax, and pollution R\&D investment costs.

Another optimization model for a manufacturing system under the environmental constraint was proposed in [28], in which production, TBM, and emission rates were determined as decision variables to minimize total cost. It was claimed that, among the related previous studies, the effect of system deterioration on the emission was addressed only in their paper; therefore, maintenance activities were considered in detail to reduce the effects of degradation.

As a related work, in [29], an ecological production and TBM policy for a deteriorating manufacturing system under carbon tax was proposed. The system subcontracts satisfy random demand and decrease the carbon tax to another deteriorating system. The objective is to determine the optimal number of PM tasks and production rates in order to minimize total maintenance, production, and carbon emission costs. They did not consider the environment (reducing the amount of carbon emission) and merely focused on minimizing the penalty costs related to their own system. In fact, the whole system can release the same carbon amount because the subcontractor generates carbon emission, too.

In [30], spare parts concept was added to previous environment-oriented production-maintenance models. In fact, in their study, environmental effects of applying new or used spare parts during corrective or PM were considered through minimizing carbon emissions and global cost of spare parts' lifetime.

Moreover, in this field, in [31], a mathematical framework was used to propose an optimal production and TBM strategy that determines both maintenance and production elements (number of maintenance activities and production rate) in order to minimize total production, maintenance, inventory, and pollution $\left(\mathrm{CO}_{2}\right.$ related $)$ costs for a deteriorating manufacturing system.

Finally, in another recent work, in [32], a sustainable TBM optimization model was proposed in which social costs (social impacts of injury caused by failures) and environmental and conventional maintenance costs were minimized. Moreover, in addition to the waste and emission generation, spare parts concept was considered in terms of sustainability.

The papers mentioned above all belong to the first category, i.e., TBM-related studies. Whilst respecting the usefulness of such studies, CBSM can be more valuable if it is applicable to the system because of the CBM advantages discussed before and those to be presented in the following.

Considering the fact that one of the maintenance operations to provide a sustainable performance of equipment is the optimization of technical conditions with respect to plant performance target [33], the condition optimization with CBM has a key role in implementing sustainable maintenance.

In addition, CBM is the most appropriate method to pursue when it is technically possible because it allows monitoring the degradation level and the resulting environmental damage in order to take appropriate preventive actions and limit the risk of penalties [34].

Despite the recommendations of previous reviewers for performing CBM studies considering sustainability factors, only few such studies have been published in recent years, some of which will be presented in the group of CBM-related studies.

A CBM model for cost minimization was presented in [35], where the objective function was aimed at reducing the replacement, maintenance, and environmental penalty costs; in addition, the decision variable was only the inspection time. In the mentioned model, when inspection reveals a given degradation level (from which the environment begins to deteriorate), the system must be replaced; otherwise, it must be allowed to continue. The authors applied the Nelder-Mead method as an optimization method to solve the problem.

Authors in [34] proposed a CMB model considering two threshold levels related to the amount of environmental damage: a critical one (which yields a significant penalty when it exceeds the threshold level) and a lower one. Herein, the threshold levels and the inspection sequence were considered to be decision variables in their work, and the total expected cost per time unit over an infinite time horizon was the objective function.

A deteriorating manufacturing system with 01 states that generated harmful emissions, which 
subcontracted to a new plant with clean production technology, was studied in [36]. The authors presented a mathematical model for minimizing the total cost of the system including inventory, backlog, production, emission, and subcontracting costs to determine the production and subcontracting rates and emission level (no maintenance-related decision variables) while including maintenance considerations in constraints.

Further, authors in [37] added the energy consumption factor to the previous CBM optimization models by considering sustainability. Their work included two aspects of sustainability: $\mathrm{CO}_{2}$ emission and energy consumption. Their proposed objective function involved the minimization of total cost, and decision variables included optimal $\mathrm{CO}_{2}$ emission and energy consumption thresholds besides the periodic inspection intervals.

The papers mentioned above belong to the second category (CBM-related studies), where decision variables are mostly related to periodic inspection rather than the maintenance optimal policy. Moreover, the aforementioned papers have not considered the assumption that a system as partially observable can usually make the model more difficult and closer to real world. Further to that, other factors of sustainability such as defective and non-recyclable products, useless replaced parts, scrapped items, and generally toxic material and waste emission are not considered.

The present paper aims to propose an optimal maintenance policy with environmental considerations that optimizes both maintenance actions and nonperiodic inspections in the context of partially observable systems. On the importance of partially observable systems, authors in a recent survey studied CBM optimization models for stochastically deteriorating systems and considered the popularity of CBM to be strongly dependent on the development of the stochastic deterioration models, which are properly used for partially observable systems [38]. In [39], a two-part study was done to highlight the advantages of applying Partially Observable Markov Decision Process (POMDP), as a stochastic control technique, for optimizing inspection and maintenance policies by applying stochastic models. The authors proposed an optimal policy for the cost-minimization CBM model in civil engineering structures (structural maintenance) with discrete-state deterioration based on a POMDP framework. They used a point-based value iteration solver (Perseus) and, also, two simpler approximate solvers based on Modified Dynamic Programming (MDPs) to solve the problem. They illustrated that the Perseus method is more efficient for this type of applications and larger state-space problems.

The present study relies on the previously reviewed works with respect to the CBM modeling approach and significantly extends it to sustainability- oriented manufacturing systems (not a structure or infrastructure) by a more rigorous, systematic formation of maintenance actions in the POMDP context. This study focuses on CBSM to present an optimization model that takes sustainability considerations and CBM goals simultaneously into account. The problem is modeled separately for four maintenance actions including keep, regular maintenance (minor repair), overhaul (major repair), and replace, with terms related to sustainability formulations. Different levels of repair can be defined between minor and major repairs, as required. $\mathrm{CO}_{2}$ and waste emissions as some of the most important factors of sustainability are included in the formulation of each action, depending on the specific situations. Other assumptions and conditions of the model will be explained in detail subsequently.

Stochastic deterioration and partial observation are two important assumptions in modeling the system; therefore, deteriorating and observation processes are both formulated by probability matrixes that represent the stochastic nature of the system.

The model is solved in both finite and infinite horizons. Perseus algorithm has been applied to solve the model approximately; besides, the problem is exactly solved with the traditional Incremental Pruning (IP) method and Accelerated Vector Pruning (AVP) method, as the current exact and fast algorithm. The objective of this work is to propose an optimal maintenance policy (including replacement, overhaul, regular repair, keep, and non-periodic inspections) as a sequence of decisions, executable by the agent based on the observations of monitors in order to achieve the system's goal (profit maximization besides the sustainability considerations) in the best possible way.

In the rest of this study, Section 2 presents the POMDP model of the CBSM problem completely, accompanied by environmental considerations in the formulation. Section 3 explains the estimated and exact value iteration algorithms, Perseus and AVP. Section 4 reports detailed computational results and concluding remarks.

\section{CBSM statement and POMDP formulation}

The CBSM problem under consideration is first described in Subsection 2.1; then, it is formulated in the POMDP framework in Subsection 2.2.

\subsection{Problem description}

Consider a CBM problem that determines the optimal maintenance/inspection policy (including the best sequence of actions and action times, monitoring, and non-periodic inspections types and intervals) by considering sustainability aspects (such as Green House Gas (GHG) and waste emission) in order to maximize 
the profit of a partially observable, stochastically deteriorating manufacturing system with uncertain action effects.

Some of the assumptions of the problem are presented as follows:

- System state is partially observable, which is monitored by several monitoring tools;

- "Keep" action in each period shows that the system should continue its manufacturing without any interference. After "keep" action, the state of the system usually cannot be improved;

- In the "regular repair" action, some routine repair works (such as resetting, lubricating, cleaning, etc.) are done for the system without stopping it. After the "regular repair", the state of the system can be improved;

- "Overhaul" action stops the manufacturing of the system and inspects and completely repairs it. In this action, the true state of the system can be observed (probability vector is $e_{j}$ );

- After "replacement" action, the system state is the state of a new system (probability vector $e_{1}$ );

- Deterioration of the system follows a stochastic process;

- Companies have many opportunities for producing environment-friendly productions that lead to gaining higher profits from environment-sensitive customers [40]. For this reason, customers' demands are considered environment sensitive and dependent on the system state in this work.

\subsection{Model formulation}

The CBSM problem is formulated in a POMDP framework in the following. In Subsection 2.2.1, inputs of the model will be introduced and illustrated in detail. In Subsection 2.2.2, first, a total schema of the model, as well as its parameters and decision variable, will be presented specifically and, then, each term of the model will be described completely.

\subsubsection{Inputs}

- $S$ : A finite set of possible states of the system $S=$ $\{1, \ldots, i, \ldots, j, \ldots, n\}$, in which $i$ denotes the system deterioration degree (1 shows "as new" system state and other numbers are sorted in ascending order to denote more deteriorated system states).

- $X$ : Real state of the system whose value comes from set $S$.

- $\Pi=\left\{\pi_{1}, \ldots, \pi_{i}, \ldots, \pi_{n}\right\}$ : The probability vector of the primitive state. The system states are random variables because of the stochastic behavior of the system that leads the model to be closer to the practical situations; moreover, since the system state is non-observable, the exact state is not available. Note that the summation of all probabilities is equal to one.

$$
\sum_{i=1}^{n} \pi_{i}=1 \text { and } 0 \leq \pi_{i} \leq 1 \text { for any } i
$$

- $V(\Pi)$ : Optimal expected total profit function over infinite horizon when the current state is $\Pi$.

- $C_{i}$ : Unit cost of the manufacturing process when the system works in state $i$ (including raw material costs, energy consuming cost, personnel hires, rent of production place, defective products, etc.). Therefore, greater deterioration (larger $i$ index) leads to increasing manufacturing cost. In other words, $C_{i}$ is an increasing function of $i$.

- $D_{i}$ : Customers' demands for product manufactured when the system works in state $i$. In this paper, it is supposed that customers' demands are environment sensitive and dependent on the health condition of the manufacturing system that supplies them (see [40]).

- $R_{i}$ : Cost of replacement (the action of replacing the system with a new one) that is a non-decreasing function of $i$. Greater deterioration (larger $i$ index) leads to decreasing the monetary value of the dismantled system and, therefore, increasing the payment to get the new system. The new system price is assumed to be constant $(R)$, and the monetary value of dismantled equipment $\left(q_{i}\right)$ is a decreasing function of $i$, and $R_{i}=R-q_{i}$.

- $\alpha_{i}$ : GHG emissions factor in the manufacturing system in tons of $\mathrm{CO}_{2}$ per unit demand of product when the system works in state $i$.

- $\omega_{i}$ : Waste emissions factor in the manufacturing system in tons of waste per unit demand when system works in state $i$.

- $\varphi_{i}$ : Defective percentage of products when the system works in state $i$.

- $G_{i}$ : Recall cost of one defective product when the system works in state $i$.

- $B_{i}$ : The $i$ th markup level considered by the manufacturing system when it works in state $i$.

- $F_{G H G}\left(E C U / T C O_{2}\right)$ : GHG emission penalty factor.

- $G H G$ : Acceptable level of GHG emission.

- $F_{\text {waste }}(E C U / T$ waste): Waste emission penalty factor.

- WST: Acceptable level of waste emission.

- $W M R_{i}$ : Waste of materials (cleaning materials utilized for "regular repair" work such as lubricating and hydraulics oils) during "regular repair" action when the system's state is $i$. 
- $W O_{i}$ : Waste of useless parts generated during "overhaul" action when the system's state is $i$.

- $W M O_{i}$ : Waste of materials (special materials utilized for "major repair" work) during "overhaul" action when the system's state is $i$.

- $W R_{i}$ : Waste of useless replaced parts generated during "replacement" action when the system's state is $i$.

- $R_{i}^{\prime}$ : Cost of regular maintenance (including standard actions such as lubricating, cleaning, resetting, etc.), which is a non-decreasing function of $i$.

- $R_{i}^{\prime \prime}$ : Cost of major repair (overhaul) including the cost of replacing defective parts of the system and the cost of other necessary actions for the restoration of the system to the new one. This parameter is also a non-decreasing function of $i$.

- $\beta$ : Discount factor between 0 and 1.

- $p_{i j}$ : One-step transition probability (during one period) from state $i$ to state $j$ in a normal condition and under "keep" action (continuing production without repair or replacement). Transition probability matrix is shown as follows:

$$
P=\begin{gathered}
1 \\
2 \\
\vdots \\
i \\
\vdots \\
n
\end{gathered}\left[\begin{array}{cccccc}
1 & 2 & \ldots & j & \ldots & n \\
p_{11} & p_{12} & \ldots & p_{1 j} & \ldots & p_{1 n} \\
p_{21} & p_{22} & \ldots & p_{2 j} & \ldots & p_{2 n} \\
\vdots & \vdots & \vdots & \vdots & \vdots & \vdots \\
p_{i 1} & p_{i 2} & \ldots & p_{i j} & \ldots & p_{i n} \\
\vdots & \vdots & \vdots & \vdots & \vdots & \vdots \\
p_{n 1} & p_{n 2} & \ldots & p_{n j} & \ldots & p_{n n}
\end{array}\right] .
$$

- $p_{i j}^{\prime}$ : One-step transition probability from state $i$ to state $j$ after regular repair. The mentioned transition probability matrix is as follows:

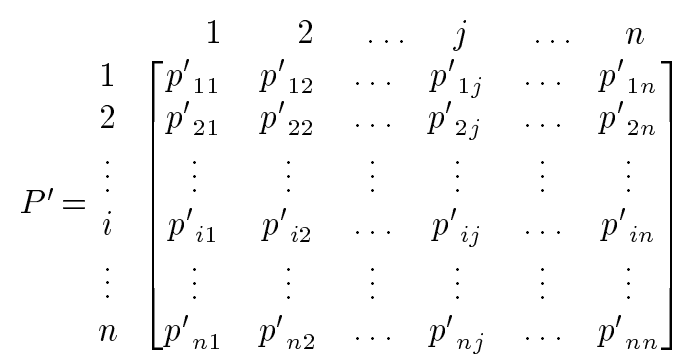

- $p_{i j}^{\prime \prime}$ : One-step transition probability from state $i$ to state $j$ after major repair, which is shown below:

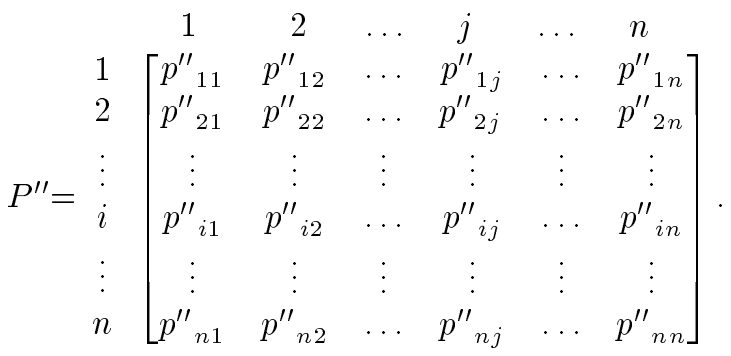

- $O=\left(o_{1}, \ldots, o_{K}, \ldots, o_{L}\right):$ Is a vector that shows the output values of monitoring in each time period. In general, for the problem under study, the system state in each time period is monitored partially by $L$ monitors. In fact, the monitors can provide relevant data to identify the real state of the system. Monitors' output is recorded as $M=$ $\left(M^{(1)}, \ldots, M^{(K)}, \ldots, M^{(L)}\right)$, where $M^{(K)}$ represents the output of the $K$ th monitor and comes from $\left\{1, \ldots, o_{K}, \ldots, m_{K}\right\}$.

- $\Gamma$ : Conditional probability matrix that elaborates on the relationships between the real state of the system and the output of monitors. This conditional probability matrix is defined by Eq. (4) as shown in Box I, where:

$$
\begin{aligned}
\gamma_{j}\left(o_{1}, \ldots, o_{L}\right) & =P(O \mid j)=\operatorname{Pr}\left(M^{(1)}\right. \\
& =o_{1}, \ldots, M^{(K)}=o_{K}, \ldots, M^{(L)} \\
& \left.=o_{L} \mid X=j\right) .
\end{aligned}
$$

$X=j$ denotes the real state of the system.

- $T(\Pi, O)$ : Updated probability vector, after observing monitors 'output information $(O)$ in a normal condition under "keep" action. In other words, $T(\Pi, O)$ reveals the secondary state (posterior probabilities) on the condition that $\Pi$ (prior probabilities) and $O$ (monitors' output) are known.

Eqs. (6)-(9) (based on Bayes' formula) are applied to calculate each element of this vector as follows:

$$
T(\Pi, O)=\left(T_{1}(\Pi, O), T_{2}(\Pi, O), \ldots, T_{n}(\Pi, O)\right),
$$

where:

$$
\Gamma=\left[\begin{array}{ccccc}
\gamma_{1}(1, \ldots, 1) & \ldots & \gamma_{1}\left(o_{1}, \ldots, o_{L}\right) & \ldots & \gamma_{1}\left(m_{1}, \ldots, m_{L}\right) \\
\vdots & \ddots & \ddots & \ddots & \vdots \\
\gamma_{j}(1, \ldots, 1) & \ldots & \gamma_{j}\left(o_{1}, \ldots, o_{L}\right) & \ldots & \gamma_{j}\left(m_{1}, \ldots, m_{L}\right) \\
\vdots & \ddots & \ddots & \ddots & \vdots \\
\gamma_{n}(1, \ldots, 1) & \ldots & \gamma_{n}\left(o_{1}, \ldots, o_{L}\right) & \ldots & \gamma_{n}\left(m_{1}, \ldots, m_{L}\right)
\end{array}\right]
$$




$$
\begin{aligned}
T_{j}(\Pi, O) & =\operatorname{Pr}(X=j \mid M=O, \Pi) \\
& =\frac{\sum_{i=1}^{n} \pi_{i} p_{i j} \gamma_{j} O}{\sum_{j=1}^{n} \sum_{i=1}^{n} \pi_{i} p_{i j} \gamma_{j} O}
\end{aligned}
$$

and:

$$
P(O \mid \Pi)=\operatorname{Pr}(M=O \mid \Pi)=\sum_{j=1}^{n} \sum_{i=1}^{n} \pi_{i} p_{i j} \gamma_{j} O,
$$

$T_{j}(\Pi, O)$ represents the probability that the system's real state is $j$ when the primary state vector (prior probabilities) is $\Pi$ and monitors report the state vector $O$. In fact, monitors' output is used to evaluate the values of $T_{j}$ to make a more precise prediction of the partially observable state of the system.

- $T^{\prime}(\Pi, O)$ : Updated probability vector by observing monitors 'output information $(O)$ after regular repair (posterior probabilities):

$$
\begin{aligned}
T^{\prime}(\Pi, O) & =\left(T_{1}^{\prime}(\Pi, O), T_{2}^{\prime}(\Pi, O), \ldots, T_{n}^{\prime}(\Pi, O)\right), \\
T_{j}^{\prime}(\Pi, O) & =\frac{\sum_{i=1}^{n} \pi_{i} p^{\prime}{ }_{i j} \gamma_{j} O}{\sum_{j=1}^{n} \sum_{i=1}^{n} \pi_{i} p_{i j}^{\prime} \gamma_{j O}}
\end{aligned}
$$

$T_{j}^{\prime}(\Pi, O)$ represents the probability that the system's real state is $j$ after the regular repair when the primary state vector is $\Pi$ and monitors report the state vector, $O$. In fact, monitors' output is used to obtain $T_{j}^{\prime}$ for a correct prediction of the partially observable state of the system. Here, it is necessary to consider that in case of major repairs and replacement, the real state is directly observable; thus, there is no need to use the updated probability vectors such as $T(\Pi, O)$ and $T^{\prime}(\Pi, O)$.

\subsubsection{Modeling}

In the following, a POMDP framework as a 6-tuple $(S, A, P, \Gamma, R w)$ is utilized to model the CBSM problem.

POMDP parameters include $S=\{1, \ldots, i, \ldots, j$, $\ldots, n\}$ as a set of states, $s_{t} \in S$ as the system state at time $t$, and $\Pi=\left\{\pi_{1}, \ldots, \pi_{i}, \ldots, \pi_{n}\right\}$ as the probability of primitive states. $A=\left\{a_{1}, a_{2}, \ldots, a_{t}\right\}$ shows the maintenance actions set at time $t$ including \{Keep, Regular, Overhaul, Replace $\}. \Omega$ is the maintenance policy that maps $S$ onto $A$. $P$ shows the transition probabilities matrix, and $P^{\prime}$ and $P^{\prime \prime}$ are the transition probabilities matrixes for "Regular" and "Overhaul" actions. $O$ is monitors' output including $\left(o_{1}, \ldots, o_{K}, \ldots, o_{L}\right)$, and $\Gamma$ shows conditional probabilities that display the relationship between $O$ and the real system state with members $\gamma_{j}\left(o_{1}, . ., o_{L}\right) . \quad \beta$ shows the discount factor between 0 and 1 . Finally, $R w\left(s_{t}, a_{t}\right)$ is the terminal reward that depends on the maintenance action and system state at time $t$ including $K V(\Pi), R g V(\Pi), O V(\Pi)$, and $R p V(\Pi)$ for "Keep", "Regular", "Overhaul", and "Replace", where the initial probability vector is $\Pi$.

CBSM parameters include $C_{i}, D_{i}, \alpha_{i}, \varphi_{i}, G_{i}$, $B_{i}, F_{G H G}, G H G, F_{\text {waste }}, W S T, \omega_{i}, W M R_{i}, W O_{i}$, $W M O_{i}$, and $W R_{i}$. Most POMDP and CBSM parameters mentioned in this section are introduced in Subsection 2.2.1 in detail.

Objective function and decision variable are introduced as follows: $V^{\Omega}(\Pi)$ in Eq. (11) represents the POMDP objective function that gives the expected total discounted reward over an infinite time horizon with initial state $\Pi$ and maintenance policy $\Omega$.

$$
V^{\Omega}(\Pi)=\sum_{i=0}^{\infty} \beta^{t} E\left[R w\left(s_{t}, a_{t}\right) \mid \Pi, \Omega\right] .
$$

Decision variable is the optimal maintenance policy denoted by $\Omega^{*}(\Pi)$ :

$$
\Omega^{*}(\Pi)=\arg \max _{a \in A} V^{\Omega}(\Pi),
$$

where $\Omega^{*}(\Pi)$ optimally suggests the type and time of maintenance actions and inspections that give the maximum expected reward for the initial state $\Pi$.

The optimal value of CBSM objective function in the POMDP framework is denoted by $V^{*}(\Pi)$ in Eq. (13):

$$
V^{*}(\Pi)=\max \{K V(\Pi), R g V(\Pi), O V(\Pi), R p V(\Pi)\},
$$

where $K V(\Pi), R g V(\Pi), O V(\Pi)$, and $R p V(\Pi)$ correspond to the expected rewards of "Keep", "Regular repair", "Overhaul", and "Replacement" actions, which are expressed separately in the following.

First, "Keep" action and its related terms are described in Eq. (14):

$$
\begin{aligned}
K V(\Pi)= & \sum_{i} \pi_{i} D_{i}\left(1+B_{i}\right) C_{i} \\
& -\left\{\sum_{i} \pi_{i} D_{i} C_{i}+\sum_{i} \pi_{i} \varphi_{i} D_{i} G_{i}\right\} \\
& -\left\{F_{G H G}\left(\sum_{i} \pi_{i} \alpha_{i}-G H G\right)\right. \\
& \left.+F_{\text {waste }}\left(\sum_{i} \pi_{i} \omega_{i} D_{i}-W S T\right)\right\} \\
& +\beta \sum_{o_{1}}^{m_{1}} \ldots \sum_{o_{L}}^{m_{L}}\left(\sum_{j} \sum_{i} \pi_{i} p_{i j} \gamma_{j} O\right) V(T(\Pi, O)) .
\end{aligned}
$$


Eq. (14) is related to "keep" action and shows the expected total discounted profit of the system when the maintenance action is in the "keep" mode in the current period. Manufacturing continues with this maintenance action; therefore, the first term in Eq. (14), $\sum \pi_{i} D_{i}\left(1+B_{i}\right) C_{i}$, shows the total income earned from supplying customers' demands. For a more detailed study on such formulations of earned profit, see [41]. Terms two and three $\left(\sum_{i} \pi_{i} D_{i} C_{i}\right.$ and $\left.\sum_{i} \pi_{i} \varphi_{i} D_{i} G_{i}\right)$ show production and recall costs. Terms four and five $\left(F_{G H G}\left(\sum_{i} \pi_{i} \alpha_{i}-G H G\right)\right.$ and $F_{\text {waste }}\left(\sum_{i} \pi_{i} \omega_{i} D_{i}-\right.$ $W S T)$ ) show GHG and waste emission penalties for "keep", respectively. Note that the penalty term is different in each maintenance action. Term $\operatorname{six}\left(\beta \sum_{o_{1}}^{m_{1}} \ldots \sum_{o_{L}}^{m_{L}}\left(\sum_{j} \sum_{i} \pi_{i} p_{i j} \gamma_{j O}\right) V(T(\Pi, O))\right)$ shows the summation of the expected total discounted profit for periods after the current period and that of "keep" that remains.

In the following, "Regular repair" and its related terms are described in Eq. (15):

$$
\begin{aligned}
R g V(\Pi)= & \sum_{i} \pi_{i} D_{i}\left(1+B_{i}\right) C_{i} \\
& -\left\{\sum_{i} \pi_{i} D_{i} C_{i}+\sum_{i} \pi_{i} \varphi_{i} D_{i} G_{i}+\sum_{i} \pi_{i} R_{i}^{\prime}\right\} \\
& -\left\{F_{G H G}\left(\sum_{i} \pi_{i} \alpha_{i}-G H G\right)\right. \\
& \left.+F_{\text {waste }}\left(\sum_{i} \pi_{i} \omega_{i} D_{i}+W M R_{i}\right)-W S T\right\} \\
& +\beta \sum_{o_{1}=1}^{m_{1}} \ldots \sum_{o_{L}=1}^{m_{L}}\left(\sum_{j} \sum_{i} \pi_{i} p^{\prime}{ }_{i j} \gamma_{j} O\right) \\
& V\left(T^{\prime}(\Pi, O)\right) .
\end{aligned}
$$

Eq. (15) shows the expected total discounted profit of the system when, in the current period, maintenance action is "regular repair". Manufacturing continues under this maintenance action; therefore, the first term in Eq. (15), $\sum_{i} \pi_{i} D_{i}\left(1+B_{i}\right) C_{i}$, shows the total income earned from supplying customers' demands. Terms two, three, and four in Eq. (15) $\left(\sum_{i} \pi_{i} D_{i} C_{i}, \sum_{i} \pi_{i} \varphi_{i} D_{i} G_{i}\right.$, and $\left.\sum_{i} \pi_{i} R_{i}^{\prime}\right)$ are related to production, recall, and regular repair costs, respectively. Terms five and six in Eq. (15) $\left(F_{G H G}\left(\sum_{i} \pi_{i} \alpha_{i}-G H G\right)\right.$ and $F_{\text {waste }}\left(\sum_{i} \pi_{i} \omega_{i} D_{i}+\right.$ $\left.W M R_{i}\right)^{i}-W S T$ ) show GHG and waste emission penalties for "Regular repair", respectively. Term seven in
Eq. (15) $\left(\beta \sum_{o_{1}=1}^{m_{1}} \ldots \sum_{o_{L}=1}^{m_{L}}\left(\sum_{j} \sum_{i} \pi_{i} p^{\prime}{ }_{i j} \gamma_{j O}\right) V\left(T^{\prime}(\Pi, O)\right)\right)$ shows the summation of the expected total discounted profit of the remaining periods after the current period and that of "Regular repair". In the following, "Overhaul" action and its related terms will be described in Eq. (16):

$$
\begin{aligned}
O V(\Pi)= & -\sum_{i} \pi_{i} R_{i}^{\prime \prime} \\
& -F_{\text {waste }}\left\{\sum_{i} \pi_{i}\left(W O_{i}+M W O_{i}\right)-W S T\right\} \\
& +\beta \sum_{j} \sum_{i} \pi_{i} p^{\prime \prime}{ }_{i j} V\left(e_{j}\right) .
\end{aligned}
$$

Eq. (16) shows the expected total discounted profit of the system when the maintenance action is "Overhaul" in the current period. Manufacturing stops under this maintenance action; therefore, terms one and two in Eq. (16) $\left(\sum_{i} \pi_{i} R^{\prime \prime}{ }_{i}\right.$ and $F_{\text {waste }}\left\{\sum_{i} \pi_{i}\left(W O_{i}+W M O_{i}\right)-\right.$ $W S T\}$ ) that are related to the current period are negative. The former shows the mean overhaul cost and the latter shows waste emission penalty of "Overhaul". Note that, in "Overhaul", there are not any manufacturing operations; thus, recall cost and GHG penalty related terms have been omitted. Term three in Eq. (16) $\left(\beta \sum_{j} \sum_{i} \pi_{i} p_{i j}^{\prime \prime} V\left(e_{j}\right)\right)$ shows the expected total discounted profit for periods that remain after this current period with "Overhaul". In the following, "Replacement" action and its related terms will be described in Eq. (17):

$$
\begin{aligned}
R p V(\Pi) & =-\sum_{i} \pi_{i} R_{i}-F_{\text {waste }}\left(\sum_{i} \pi_{i} W R_{i}-W S T\right) \\
& +\beta V\left(e_{1}\right) .
\end{aligned}
$$

Eq. (17) shows the expected total discounted profit of the system when the maintenance action is "Replace" in the current period. Manufacturing stops in this maintenance action; therefore, Terms one and two in Eq. (17) $\left(\sum_{i} \pi_{i} R_{i}\right.$ and $\left.F_{\text {waste }}\left(\sum_{i} \pi_{i} W R_{i}-W S T\right)\right)$, which are related to the current period, are negative. The prior shows mean replacement cost, and the latter shows the waste emission penalty for "Replace". Note that, in this maintenance action, there are not any manufacturing operations; therefore, recall cost and GHG penalty-related terms have been omitted. Term three in Eq. (17) $\left(\beta V\left(e_{1}\right)\right)$ shows the expected total discounted profit for periods that remain after the current period with "Replace".

In the next section, the CBSM model studied in this section will be solved efficiently by 4 methods: 
Standard Dynamic Programming (SDP), Modified Dynamic Programming (MDP), IP, and AVP. Solution approaches are illustrated, and related algorithms and their flowcharts are presented.

\section{Solution approach}

In this section, solving the CBSM model, presented in the previous section, will be studied.

In general, POMDP solving methods can be divided into two main categories: optimal solving methods and near-optimal and approximate methods. Some of these methods are applied to solve the presented CBSM model in this paper: SDP method (a basic traditional optimal method and MDP method (a recent version of it), IP method (a more recent and popular optimal method) and AVP (a very recent version of it), and Perseus (one of the most popular approximate methods).

Each of the mentioned solving methods will be described further and compared with each other in the following.

For the first time, the concept of maintenance in the POMDP framework was studied in [42]. The concept is known to be a proper method to apply to partially observable systems under uncertainty [43] and has been considered by several studies; however, the optimal solving mode of it was abandoned long ago (until 2010) because of the complexity and difficulty of data acquisition.

SDP as a traditional optimal solving method, which is applied to solve the CBSM problem in this paper, can present an optimal solution to small-sized problems, but it is unable to solve medium and large POMDPs. On the other hand, MDP as a revised version of SDP can solve small and medium problems, but it cannot solve large problems.

Therefore, near-optimal and approximate methods were proposed for solving POMDPs, some of the most important of which exist in the papers such as [44] and [45].

Among the popular approximate methods, Perseus method, described as an approximate pointbased solver in [45], will be used and evaluated in the next section for solving the mentioned CBSM model, in which there are some features that make it even more complicated than simple POMDP to solve. Unlike SDP and MDP, Perseus can solve large-scale CBSM problems in a desirable time span, but the solution is approximate and not exact.

To present an optimal and exact solution to the POMDP problems in a desirable amount of time, some recent papers were proposed in [46-51].

The application of IP variations as an exact method has become increasingly popular in recent works because of their efficiency in solving POMDPs.
Pruning methods use a piecewise-linear and convex representation of the value function that can be specified by a unique set of vectors and often aim to remove nonnecessary vectors. In [51], as the most recent work, a new variant of IP method called AVP is introduced for the exact solving of large-scale POMDPs so as to improve the performance of existing pruning methods and make the proposed method the fastest of its kind for POMDPs. The AVP method will be applied for optimally solving the CBSM model, too.

In the remainder of this section, SDP and MDP methods will be explained in Subsection 3.1, Perseus with its flowchart will be described in Subsection 3.2, and IP method and AVP will be illustrated with their CBSM-related flowcharts in Subsection 3.3. Finally, Perseus and AVP methods as the most effective estimating and exact methods will be compared with each other.

\subsection{Standard and modified dynamic programming}

CBSM problem has been solved using SDP as a traditional, exact POMDP solving method in the finite horizon (see [52]). A backward profit maximization recursive algorithm, which can solve problems of DP kind, is employed and implemented using visual basic and $\mathrm{C}++$ solvers. Computational results show that it is difficult to apply this method to solve mediumand large-scale POMDP problems. Limitations of the classical DP are well known because of its long time and high memory consumption during the computation.

In the present paper, some techniques in $\mathrm{C}++$ programming are applied to increase the system's memory temporarily and reduce DP's computational limitations. These methods include memory management techniques, heap memory management methods, and a private heap for dynamic memory allocation and handling memory blocks [53]. This method is named MDP, the application of which necessitates allocating a greater volume of memory to processing and ensures a shorter computational duration. Computational results of solving CBSM model in the finite horizon by standard and modified DP methods and comparing them with other methods will be presented in Table 1 in Section 4.

In the next section, Perseus method that can overcome the limitations of DP and MDP methods in solving large-scale problems will be described.

\subsection{Perseus method}

Since exact methods are usually not suitable for solving large-scale and complicated POMDPs, approximate methods like Perseus have gained the upper hand and become popular [54].

Perseus method is applied to solve the proposed CBSM model efficiently; it is a randomized Point- 
Table 1. Computational time of solving Condition-Based Sustainable Maintenance (CBSM) model by Standard and Modified DPs (3 states and 2 monitors in the finite horizon).

\begin{tabular}{|c|c|c|c|c|c|c|}
\hline \multirow[b]{3}{*}{$\mathbf{N}$} & \multirow{3}{*}{$\begin{array}{l}\text { \# of stages or } \\
\text { horizon length }\end{array}$} & \multirow{3}{*}{$\begin{array}{c}\text { Initial } \\
\text { probability vector }\end{array}$} & \multicolumn{4}{|c|}{ Computation time (s) } \\
\hline & & & \multicolumn{2}{|c|}{ Standard DP } & \multicolumn{2}{|c|}{ Modified DP } \\
\hline & & & $\begin{array}{c}\text { State } \\
\text { generation }\end{array}$ & $\begin{array}{c}\text { Decision- } \\
\text { making }\end{array}$ & $\begin{array}{c}\text { State } \\
\text { generation }\end{array}$ & $\begin{array}{c}\text { Decision- } \\
\text { making }\end{array}$ \\
\hline 1 & 5 & $(1,0,0)$ & 59 & 78 & 0.5 & 1.5 \\
\hline 2 & 7 & $(1,0,0)$ & 610 & 720 & 1.5 & 2 \\
\hline 3 & 10 & $(1,0,0)$ & $\times$ & $x$ & 10 & 15 \\
\hline 4 & 20 & $(1,0,0)$ & $\times$ & $\times$ & 203 & 224 \\
\hline 5 & 25 & $(1,0,0)$ & $\times$ & $\times$ & $\times$ & $\times$ \\
\hline 6 & 5 & $(0.8,0.15,0.05)$ & 63 & 85 & 1 & 1.5 \\
\hline 7 & 7 & $(0.8,0.15,0.05)$ & 602 & 732 & 2 & 3 \\
\hline 8 & 10 & $(0.8,0.15,0.05)$ & $x$ & $x$ & 11 & 19 \\
\hline 9 & 20 & $(0.8,0.15,0.05)$ & $\times$ & $x$ & 240 & 271 \\
\hline 10 & 25 & $(0.8,0.15,0.05)$ & $\times$ & $\times$ & $\times$ & $\times$ \\
\hline 11 & 5 & $(0,1,0)$ & 62 & 84 & 1 & 1.5 \\
\hline 12 & 7 & $(0,1,0)$ & 667 & 722 & 3 & 3 \\
\hline 13 & 10 & $(0,1,0)$ & $\times$ & $\times$ & 10 & 15 \\
\hline 14 & 20 & $(0,1,0)$ & $\times$ & $\times$ & 258 & 277 \\
\hline 15 & 25 & $(0,1,0)$ & $x$ & $x$ & $x$ & $x$ \\
\hline 16 & 6 & $(0.2,0.3,0.5)$ & 619 & 705 & 0.9 & 1.3 \\
\hline 17 & 7 & $(0.2,0.3,0.5)$ & $\times$ & $x$ & 2 & 3 \\
\hline 18 & 10 & $(0.2,0.3,0.5)$ & $\times$ & $x$ & 14 & 16 \\
\hline 19 & 20 & $(0.2,0.3,0.5)$ & $x$ & $x$ & 246 & 261 \\
\hline 20 & 25 & $(0.2,0.3,0.5)$ & $x$ & $x$ & $x$ & $x$ \\
\hline 21 & 6 & $(0,0,1)$ & 590 & 682 & 1.2 & 1.8 \\
\hline 22 & 7 & $(0,0,1)$ & $x$ & $\times$ & 2 & 3 \\
\hline 23 & 10 & $(0,0,1)$ & $\times$ & $\times$ & 11 & 14 \\
\hline 24 & 20 & $(0,0,1)$ & $x$ & $x$ & 235 & 265 \\
\hline 25 & 25 & $(0,0,1)$ & $x$ & $\times$ & $x$ & $x$ \\
\hline
\end{tabular}

Based Value Iteration (PBVI) algorithm that first collects a quite large set of belief points from the belief space, which remains fixed until the end of the algorithm. By simply choosing random actions and observations to create trajectories via the belief space, $B$ will be built. Next, the algorithm computes the initial value function, which is considered as an approximated lower bound, commonly including a single $\alpha$-vector with all components calculated by $\frac{1}{1-\gamma} \min _{s, a} R(s, a)$. In the next step, backup stages will begin and continue until the end of algorithm, where a belief point $b$ will be chosen randomly in each stage from $B$ to calculate a related value and $\alpha$-vector. To briefly explain the backup stages, a flowchart of the algorithm is shown in Figure 1.

In the last step of the flowchart, the convergence condition is satisfied if the absolute value difference in two successive iterations is below a defined tolerance threshold (0.001), or the running time exceeds a defined limit (2000 seconds), as described in Section 3.

Moreover, the computational results of solving the CBSM model by Perseus method and the comparison of the former results and the results of other methods are presented in Table 2 in Section 4. 


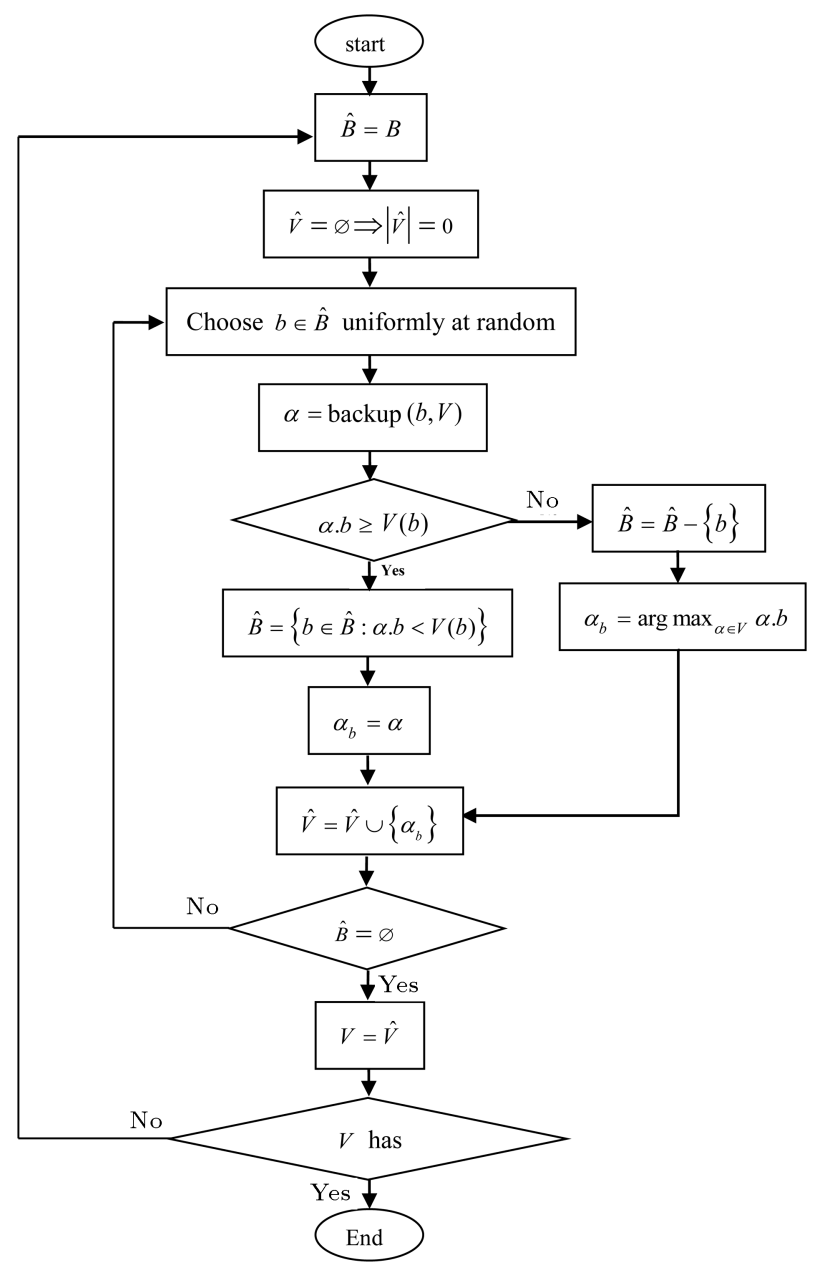

Figure 1. Flowchart of the Perseus algorithm.

In the next section, a new AVP method that can solve large-scale problems exactly will be described.

\subsection{Accelerated Vector Pruning (AVP) and Incremental Pruning (IP) methods}

As mentioned before, the application of AVP for some optimal POMDP solvers is a recently proposed procedure that speeds up the existing pruning algorithms and leads to the exact solving of large-scale POMDP problems in a desirable amount of time. Generally, pruning methods work based on the fact that the value function in POMDPs is piecewise linear and convex [55], which can be specified by using a unique set of vectors with a minimum size. Pruning algorithms often aim to remove unnecessary or dominated vectors from the value function; otherwise, the number of possible vectors will increase quickly and can lead to slowing down the process of finding an optimal policy in the steps of the exact value iteration algorithm.

This property is used for representing the value function by a finite set of $|S|$-dimensional vectors, $\Upsilon=\left\{\nu^{0}, \nu^{1}, \ldots, \nu^{k}\right\}$, in which the belief point $b$ has a value of $V(b)=\max _{\nu^{n} \in \Upsilon} b . \nu^{n}$, where "." denotes
Table 2. Computational results of solving Condition-Based Sustainable Maintenance (CBSM) model by Perseus, Incremental Pruning (IP), and Accelerated Vector Pruning (AVP) methods in the infinite horizon, Initial probability vector $(0.8,0.15,0.05)$.

\begin{tabular}{|c|c|c|c|c|c|c|}
\hline \multirow{3}{*}{$\mathbf{N}$} & \multirow{3}{*}{$|O|$} & \multirow{3}{*}{$|\boldsymbol{A}|$} & \multicolumn{4}{|c|}{ Computation time (s) } \\
\hline & & & \multicolumn{2}{|c|}{ Perseus } & \multirow[b]{2}{*}{ IP } & \multirow[b]{2}{*}{ AVP } \\
\hline & & & Error (\%) & Time & & \\
\hline 1 & 2 & 4 & 0 & 0.2 & 31 & 27 \\
\hline 2 & 3 & 4 & 0 & 19 & 135 & 48 \\
\hline 3 & 4 & 4 & $5.3 \mathrm{E}-8$ & 32 & 228 & 170 \\
\hline 4 & 6 & 4 & $9.6 \mathrm{E}-7$ & 61 & 642 & 345 \\
\hline 5 & 2 & 4 & $5.8 \mathrm{E}-8$ & 8 & 56 & 49 \\
\hline 6 & 3 & 4 & $9.6 \mathrm{E}-7$ & 21 & 202 & 119 \\
\hline 7 & 3 & 6 & $9.5 \mathrm{E}-6$ & 76 & 693.5 & 468 \\
\hline 8 & 3 & 8 & $9.0 \mathrm{E}-6$ & 100 & 1123 & 900 \\
\hline 9 & 4 & 4 & 0 & 54 & 499 & 290 \\
\hline 10 & 5 & 3 & $9.7 \mathrm{E}-7$ & 75 & 783 & 446 \\
\hline 11 & 5 & 4 & $9.7 \mathrm{E}-7$ & 81 & 809 & 2226 \\
\hline 12 & 6 & 4 & $9.8 \mathrm{E}-6$ & 105 & 1196 & 517 \\
\hline 13 & 3 & 6 & $9.9 \mathrm{E}-6$ & 110 & 1261 & 581 \\
\hline 14 & 3 & 8 & $9.0 \mathrm{E}-6$ & 139 & 1994 & 1005 \\
\hline 15 & 5 & 3 & $9.6 \mathrm{E}-5$ & 97 & 1019 & 963 \\
\hline 16 & 5 & 4 & $9.2 \mathrm{E}-6$ & 108 & 1249 & 541 \\
\hline 17 & 6 & 4 & $9.8 \mathrm{E}-5$ & 113 & 1724 & 956 \\
\hline 18 & 2 & 4 & $9.6 \mathrm{E}-6$ & 17 & 102 & 58 \\
\hline 19 & 3 & 6 & $9.8 \mathrm{E}-6$ & 120 & 1882 & 948 \\
\hline 20 & 4 & 4 & $9.5 \mathrm{E}-6$ & 63 & 667 & 363 \\
\hline 21 & 4 & 4 & $9.6 \mathrm{E}-5$ & 98 & 1061 & 735 \\
\hline 22 & 2 & 4 & $9.2 \mathrm{E}-4$ & 34 & 255 & 89 \\
\hline 23 & 4 & 4 & $9.8 \mathrm{E}-4$ & 169 & 1525 & 949 \\
\hline 24 & 21 & 5 & $9.7 \mathrm{E}-4$ & 1048 & $\times$ & $\times$ \\
\hline 25 & 17 & 5 & $9.7 \mathrm{E}-4$ & 816 & $x$ & $x$ \\
\hline
\end{tabular}

"dot product" (for more details about this, refer to [56] or $[57])$.

The CBSM model proposed in the present paper can also be rewritten in the vector form. Based on the steps proposed in [58], first, the value function is defined as a combination of simpler value functions:

$$
V(\Pi)=\max _{a \in A} V^{a}(\Pi)
$$




$$
\begin{aligned}
& V^{a}(\Pi)=\sum_{O_{1}=1}^{m_{1}} \cdots \sum_{O_{L}=1}^{m_{L}}\left\{V^{a, \mathrm{O}}(\Pi)\right\} \\
& V^{\text {Keep }}(\Pi)=\sum_{O_{1}=1}^{m_{1}} \cdots \sum_{O_{L}=1}^{m_{L}}\left\{V^{\text {Keep }, \mathrm{O}}(\Pi)\right\} \\
& V^{\text {Regular }}(\Pi)=\sum_{O_{1}=1}^{m_{1}} \cdots \sum_{O_{L}=1}^{m_{L}}\left\{V^{\text {Regular, } \mathrm{O}}(\Pi)\right\} \\
& V^{\text {Overhaul }}(\Pi)=\sum_{O_{1}=1}^{m_{1}} \cdots \sum_{O_{L}=1}^{m_{L}}\left\{V^{\text {Overhaul,O }}(\Pi)\right\} \\
& V^{\text {Replace }}(\Pi)=\sum_{O_{1}=1}^{m_{1}} \cdots \sum_{O_{L}=1}^{m_{L}}\left\{V^{\text {Replace }, \mathrm{O}}(\Pi)\right\} .
\end{aligned}
$$

Eqs. (24)-(27) are shown in Box II. All of these functions are piecewise linear and convex that can be purged from dominated vectors.

Purged sets with minimum sizes will be shown by Purge() operator as $\Upsilon(\Pi), \Upsilon^{a}(\Pi)$, and $\Upsilon^{a}, O(\Pi)$ for $V(\Pi), V^{a}(\Pi)$, and $V^{a, O}(\Pi)$, respectively. Therefore,
Eqs. (28) to (30) can be written as follows:

$$
\begin{aligned}
& \Upsilon(\Pi)=\operatorname{Purge}\left(\cup_{a \in A} \Upsilon^{a}(\Pi)\right) \\
& \Upsilon^{a}(\Pi)=\operatorname{Purge}\left(\oplus_{o \in O} \Upsilon^{a, O}(\Pi)\right) \\
& \Upsilon^{a, O}(\Pi)=\operatorname{Purge}\left(\left\{\nu^{a, O, i} \mid \nu^{i} \in \Upsilon\right\}\right) \\
& \nu^{a, O, n}(s)=\frac{R(i, a)}{|O|}+\beta \sum_{j \in S} P(o \mid a, j) P(j \mid a, i) \nu^{n}(j)
\end{aligned}
$$

In Eq. (29), $\oplus$ is the vector-related operator and is defined for two sets $Y$ and $z$ as $Y \oplus Z=\{y+z \mid y \in$ $Y, z \in Z\}$.

Dominated vectors in some mathematical methods are detected by solving a number of Linear Programming (LP)problems, which is usually a timeconsuming operation. In the present paper, two different LP methods are considered: a classic LP algorithm (related to IP method) and the AVP LP algorithm. Figure 2 shows a flowchart that summarizes the steps of the pruning algorithms proposed in [59]. Sections related to LP problem solving are in bold. Note that the $<_{\text {lex }}$ in the flowchart is lexicographic ordering, and its application to this algorithm is explicit in [60].

As mentioned before, AVP is a new variant of the IP method that uses Benders decomposition approach

$$
\begin{aligned}
& V^{\mathrm{Keep}, \mathrm{O}}(\Pi)=\frac{1}{|O|}\left\{\begin{array}{l}
\sum_{i} \pi_{i} D_{i}\left(1+B_{i}\right) C_{i}-\left\{\sum_{i} \pi_{i} D_{i} C_{i}+\sum_{i} \pi_{i} \varphi_{i} D_{i} G_{i}\right\}- \\
\left\{F_{G H G}\left(\sum_{i} \pi_{i} \alpha_{i}-G H G\right)+F_{\text {waste }}\left(\sum_{i} \pi_{i} \omega_{i} D_{i}-W S T\right)\right\}
\end{array}\right\} \\
& +\beta\left(\sum_{j} \sum_{i} \pi_{i} p_{i j} \gamma_{j} O\right) V(T(\Pi, O)) \\
& V^{\text {Regular }, \mathrm{O}}(\Pi)=\frac{1}{|O|}\left\{\begin{array}{l}
\sum_{i} \pi_{i} D_{i}\left(1+B_{i}\right) C_{i}-\left\{\sum_{i} \pi_{i} D_{i} C_{i}+\sum_{i} \pi_{i} \varphi_{i} D_{i} G_{i}+\sum_{i} \pi_{i} R_{i}^{\prime}\right\}- \\
\left.\left\{F_{G H G}\left(\sum_{i} \pi_{i} \alpha_{i}-G H G\right)+F_{\text {waste }}\left(\sum_{i} \pi_{i} \omega_{i} D_{i}-W M R_{i}\right)-W S T\right)\right\}
\end{array}\right\} \\
& +\beta\left(\sum_{j} \sum_{i} \pi_{i} p_{i j}^{\prime} \gamma_{j O}\right) V\left(T^{\prime}(\Pi, O)\right) \\
& V^{\text {Overhaul,O }}(\Pi)=\frac{1}{|O|}\left\{-\sum_{i} \pi_{i}{R^{\prime \prime}}_{i}-F_{\text {waste }}\left\{\sum_{i} \pi_{i}\left(W O_{i}+W M O_{i}\right)-W S T\right\}+\beta\left(\sum_{j} \sum_{i} \pi_{i} p^{\prime \prime}{ }_{i j} V\left(e_{j}\right)\right)\right\}, \\
& V^{\text {Replace, } \mathrm{O}}(\Pi)=\frac{1}{|O|}\left\{-\sum_{i} \pi_{i} R_{i}-F_{\text {waste }}\left(\sum_{i} \pi_{i} W R_{i}-W S T\right)+\beta V\left(e_{1}\right)\right\} .
\end{aligned}
$$




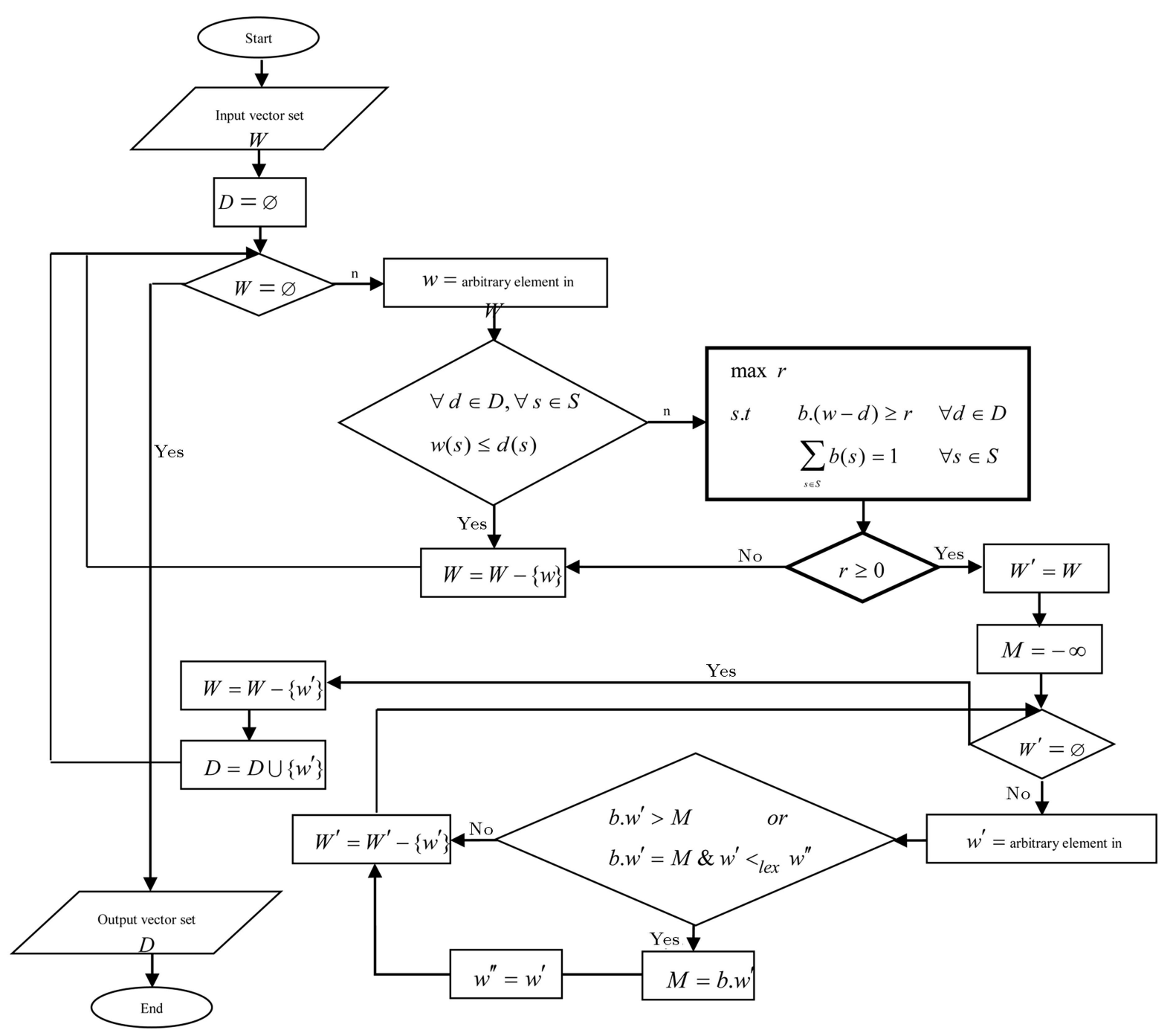

Figure 2. Flowchart of the pruning algorithm.

to accelerate the pruning procedure by decomposing linear programs to be solved among the pruning phases in the IP method. Therefore, the steps of AVP method are similar to those shown in Figure 2 (related to the IP method) and differ from those in the LP section, which must be replaced by the flowchart presented in Figure 3.

Note that all inputs, outputs, and the overall results are the same in both cases of the original LP and the decomposed LP procedures; however, the program is often faster in the second decomposed procedure because there are a small number of original LP's constraints in the decomposed LP. For more details about AVP method such as how to apply Benders decomposition to it and prove its correctness, please refer to [51]. In addition to the Perseus method illustrated in the previous section, for the CBSM model, AVP and IP methods will be applied to solve the CBSM model in value iteration algorithm, and computational results will be compared with each other. IP has been considered as the most efficient method among the pruning methods before AVP in several papers.

In the next section, computational results of solving CBSM problem with SDP, MDP, Perseus, IP, and AVP methods will be presented.

\section{Computational results and concluding remarks}

For evaluating the performance of algorithms proposed in the previous section, numerical studies are provided in this section. Algorithms such as standard DP, modified DP, Perseus, IP, and AVP were coded in visual basic, $\mathrm{C}++$, and Java languages with a $\mathrm{PC}$ featuring a $2.4 \mathrm{GHz}$ Pentium IV processor, 4GB RAM, and Windows Vista.

As mentioned earlier, exact methods, SDP and MDP, can solve small POMDPs. Therefore, the 


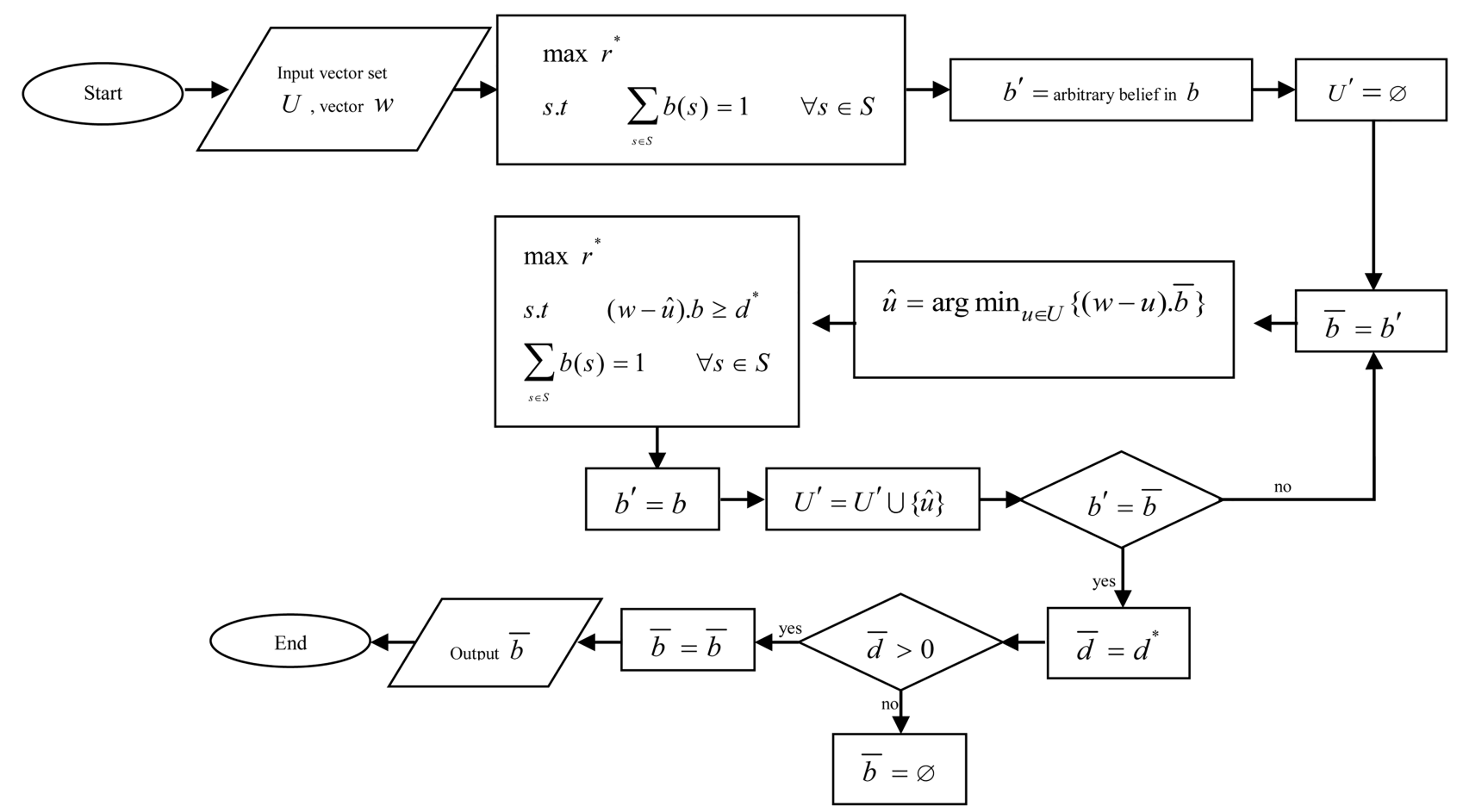

Figure 3. Flowchart of the decomposed Linear Programming (LP) part in the Accelerated Vector Pruning (AVP) algorithm.

effectiveness of these algorithms in solving such CBSM problems was examined and compared by performing a set of computational experiments including small problems with maximum 20 stages (horizon length), 3 states (healthy, medium, and broken states of the system), and 2 condition monitoring tools (for example, temperature and vibration sensors) in various primary states including $(1,0,0)$ for healthy primary states, $(0,1,0)$ for medium primary states, $(0,0,1)$ for broken primary states, and $(0.2,0.3,0.5)$ and $(0.8,0.15,0.05)$ for fairly broken and fairly healthy primary states, as shown in Table 1. Memory limitation is observed in more than 20 stages for 3 -state and 2-sensor problems, as shown by $\times$ in Table 1 .

Infinite horizon CBSM problems can be solved by Perseus, IP, and AVP methods, and the computational results of comparing their solution time with each other are presented in Table 2 for the probability vector of primitive states $(0.8,0.15,0.05)$. These probabilities show that the underlying system is in a healthy state with an $80 \%$ probability level at the start of the maintenance process. This means that, under such a condition, maintenance planning will be done for a system that is most likely healthy at the start of the process to represent an intuitive position. From Table 2, it can be seen that the proposed AVP algorithm is very successful in achieving exact solutions in a reasonable amount of time (all less than 2300 seconds), which indicates the efficiency of the AVP method in solving such CBSM problems. Moreover, Perseus is very successful in proposing high-quality solutions with fewer errors, all less than $0.01 \%$.

A summary of sensitivity analysis and managerial remarks is reported in Table 3 and Figures 4 to 8 .

Table 1 compares the computational time taken to solve finite-horizon small and medium CBSM problems by SDP and MDP. The initial probability vector is considered for some of the following intuitive states: the completely broken, the completely healthy, the medium state, the likely healthy $(80 \%)$, and the likely broken (50\%). SDP can only solve the instances until 7 stages. Symbol " $\times$ " is used in this table to show "memory limitation". As shown in Table 1, MDP is often faster than SDP and needs a lower amount of memory; however, large problems with more than 20 stages, 3 states, and 2 monitors cannot be solved

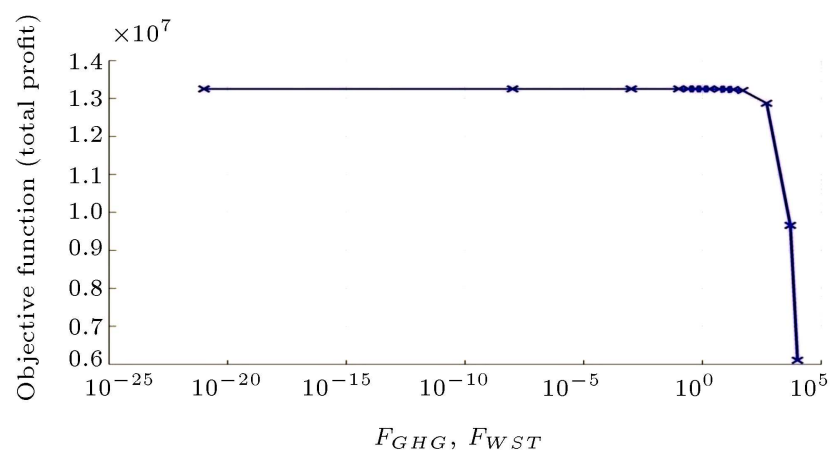

Figure 4. Sensitivity analysis of total profit for sustainability factors. 
Table 3. Sensitivity analysis of sustainability factors $F_{G H G}$ and $F_{\text {waste }}, G H G=W S T=25$.

\begin{tabular}{cccccccc}
\hline $\mathbf{N}$ & $\boldsymbol{F}_{\boldsymbol{G H G}}$ & $\boldsymbol{F}_{\text {waste }}$ & Objective function & \multicolumn{2}{c}{ Limit violation } & \multicolumn{2}{c}{ Penalty } \\
& & & & of GHG & of WST & of GHG & of WST \\
\hline 1 & 0 & 0 & 13249839.2 & - & - & 0 & 0 \\
2 & $1 \times 10^{-20}$ & $1 \times 10^{-20}$ & 13249839.2 & $3.28 \mathrm{E}+26$ & $1.4 \mathrm{E}+27$ & 327840 & 1397183.9 \\
3 & $1 \times 10^{-8}$ & $1 \times 10^{-8}$ & 13249839.2 & $3.28 \mathrm{E}+13$ & $1.4 \mathrm{E}+14$ & 327840 & 1397183.9 \\
4 & $1 \times 10^{-4}$ & $1 \times 10^{-4}$ & 13249838 & 1397183836.5 & 327839962.3 & 327840 & 1397183.8 \\
5 & $1 \times 10^{-1}$ & $1 \times 10^{-1}$ & 13249754 & 3278323.9 & 139717536.5 & 327832.4 & 1397175.4 \\
6 & 0.25 & 0.25 & 13249625 & 1311283.6 & 5588650.1 & 327820.9 & 1397162.5 \\
7 & 0.5 & 0.5 & 13249411 & 655603.5 & 2794282.3 & 327801.8 & 1397141.1 \\
8 & 1 & 1 & 13248984 & 327763.5 & 1397098.4 & 327763.5 & 1397098.4 \\
9 & 2 & 2 & 13248128 & 163843.5 & 698506.4 & 327687 & 1397012.8 \\
10 & 5 & 5 & 13245561 & 65491.47 & 279351.2 & 327457.4 & 1396756.1 \\
11 & 10 & 10 & 13241283 & 32707.47 & 139632.8 & 327074.7 & 1396328.3 \\
12 & 20 & 20 & 13232726 & 16315.47 & 69773.63 & 326309.3 & 1395472.6 \\
13 & 50 & 50 & 13207443 & 6480.265 & 27858.89 & 324013.3 & 1392944.3 \\
14 & 500 & 500 & 12872170.9 & 598.2358 & 2718.8 & 299117.9 & 1359417.1 \\
15 & 5000 & 5000 & 9657669 & 23.7732 & 207.5934 & 118866 & 1037966.9 \\
16 & 10000 & 10000 & 6107507 & 7.860074 & 68.29507 & 78600.74 & 6882950.7 \\
\hline
\end{tabular}

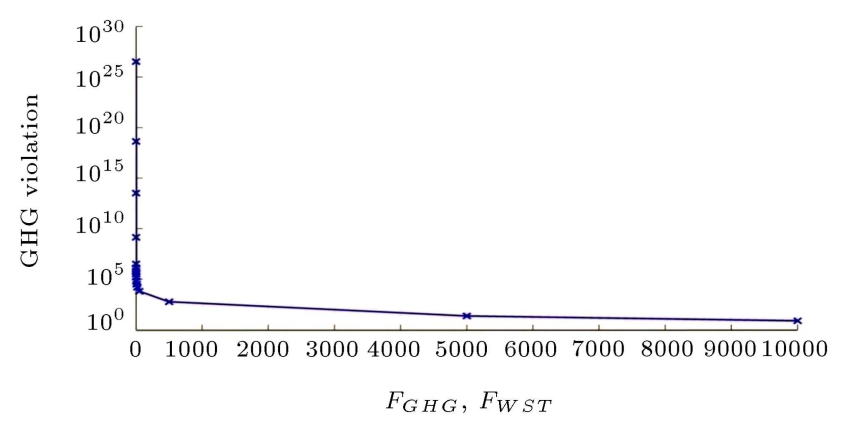

Figure 5. Sensitivity analysis of GHG violation for sustainability factors.

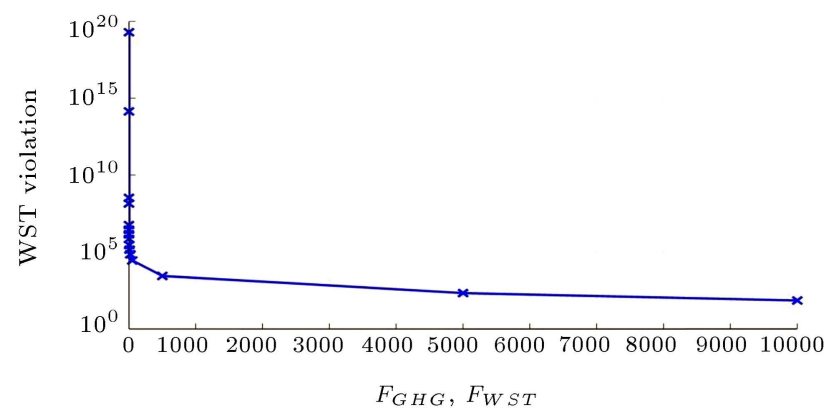

Figure 6. Sensitivity analysis of WST violation for sustainability factors.

by the method yet. In the following, more powerful computational results will be presented by applying Perseus and AVP methods in the infinite time horizon.

Table 2 shows computational results such as computation time and error percentage for solving small, medium, and large problems in the infinite time horizon by Perseus, IP, and AVP methods. Symbol $\times$ is used to show "memory limitation". It can be observed from

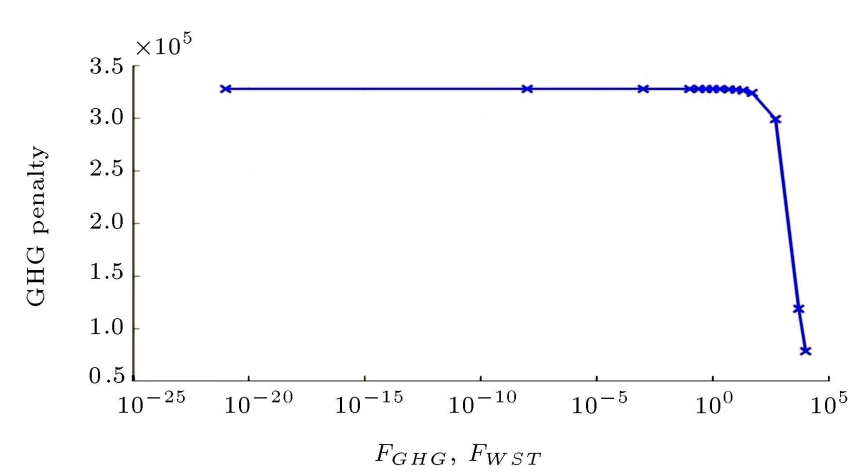

Figure 7. Sensitivity analysis of GHG penalty for sustainability factors.

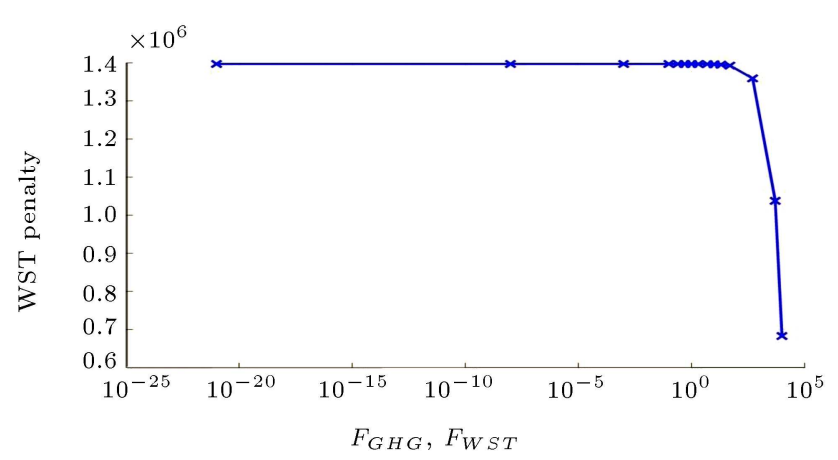

Figure 8. Sensitivity analysis of waste penalty for sustainability factors.

Table 2 that AVP improves the performance of IP in all CBSM cases and needs a lower volume of memory. It should be noted that other larger problems, such as those shown in the last two rows, could not be solved by AVP and IP; however, Perseus can solve them approximately. It is notable that in the case of pruning 
methods such as IP and AVP, problems that have initial probability vectors with fewer zeros are often more time consuming and, therefore, the intuitive initial state $(0.8,0.15,0.05)$ that shows mostly a healthy state for the system is selected to begin the maintenance procedure, as shown in this table.

Table 3 shows the objective function, GHG, and waste violation and also GHG and waste penalty variations versus sustainability factors $F_{G H G}$ and $F_{\text {waste }}$ changes. The analytical results of this table are presented in five figures in the following.

Figures 4 to 8 show the impact of changing sustainability factors $\left(F_{G H G}\right.$ and $F_{\text {waste }}$ ) on some related values (such as objective function, GHG, and waste violation and also GHG and waste penalty). Decreasing sustainability factors $\left(F_{G H G}\right.$ and $\left.F_{\text {waste }}\right)$ means easier and more flexible governmental regulations. If these penalty factors go below a certain level, manufacturing systems violate GHG and waste limitations ( $G H G$ and $W S T$ ) heavily in the exponential form (see Figures 5 and 6 ) because the assigned penalties can be controllable simply (see Figures 7 and 8 ) and there will not be any significant decrease in the manufacturing systems' total profit. This could be a serious threat to the environment.

On the other hand, increasing sustainability factors $\left(F_{G H G}\right.$ and $\left.F_{\text {waste }}\right)$ means greater stricture and tougher governmental regulations, and increasing the environment-related penalties for GHG and waste can only useful up to a certain limit, before which manufacturing systems attempt to produce less pollution to control their profit level; however, after the aforementioned set limit, the manufacturing systems cannot withstand, and there remains only one way to deal with these excessive penalties: limiting production (see Figure 8).

\section{Conclusions}

This study focused on environmental considerations in the maintenance context of partially observable, stochastically deteriorating systems to optimize both maintenance and inspection actions. The Partially Observable Markov Decision Process (POMDP) framework with a more rigorous, systematic formation of maintenance actions was applied to model sustainability-oriented manufacturing system's maintenance problem. The problem was modeled separately for four maintenance actions including keep, regular maintenance (minor repair), overhaul (major repair), and replace with terms related to sustainability formulations. $\mathrm{CO}_{2}$ and waste emissions as some of the most important factors of sustainability were considered in the formulation of each action, depending on the specific situations of it.

The model was solved for both finite and infinite horizons. Perseus algorithm was applied for solving the model approximately. Apart from that, the problem was solved by an exact method, Incremental Pruning (IP), and also with a current variant of it, Accelerated Vector Pruning (AVP) method. By comparing the computational results and analyzing them, some interesting theoretical and managerial points were obtained. The optimization of Green House Gas (GHG) and waste factors can be proposed for the future research.

\section{References}

1. Chu, C., Proth, J.M., and Wolff, P. "Predictive maintenance: The one-unit replacement model", International Journal of Production Economics, 54(3), pp. 285-295 (1998).

2. Wireman, T., Benchmarking Best Practices in Maintenance Management, Industrial Press Inc (2004).

3. Waeyenbergh, G. and Pintelon, L. "A framework for maintenance concept development", International Journal of Production Economics, 77(3), pp. 299-313 (2002).

4. Rahmati, S.H.A., Ahmadi, A., and Karimi, B. "Developing simulation based optimization mechanism for a novel stochastic reliability centered maintenance problem", Scientia Iranica, Transactions E, Industrial Engineering, 25(5), pp. 2788-2806 (2018).

5. Agustiady, T.K. and Cudney, E.A. "Total productive maintenance", Total Quality Management \& Business Excellence, pp. 1-8 (2018) .

6. Nielsen, J.S. and S $\phi$ rensen, J.D. "Computational framework for risk-based planning of inspections, maintenance and condition monitoring using discrete Bayesian networks", Structure and Infrastructure Engineering, 14(8), pp. 1082-1094 (2018).

7. Ahmad, R. and Kamaruddin, S. "An overview of timebased and condition-based maintenance in industrial application", Computers \& Industrial Engineering, 63(1), pp. 135-149 (2012).

8. Takata, S., Kirnura, F., Van Houten, F.J.A.M., et al. "Maintenance: changing role in life cycle management", CIRP Annals Manufacturing Technology, 53(2), pp. 643-655 (2004).

9. Grall, A., Bérenguer, C., and Dieulle, L. "A conditionbased maintenance policy for stochastically deteriorating systems", Reliability Engineering \& System Safety, 76(2), pp. 167-180 (2002a).

10. Han, Y. and Song, Y.H. "Condition monitoring techniques for electrical equipment-a literature survey", Power Delivery, IEEE Transactions on, 18(1), pp. 413 (2003).

11. Moya, M.C.C. "The control of the setting up of a predictive maintenance programme using a system of indicators", Omega, 32(1), pp. 57-75 (2004).

12. Jardine, A.K., Lin, D., and Banjevic, D. "A review on machinery diagnostics and prognostics implementing 
condition-based maintenance", Mechanical Systems and Signal Processing, 20(7), pp. 1483-1510 (2006).

13. Gupta, A. and Lawsirirat, C. "Strategically optimum maintenance of monitoring-enabled multi-component systems using continuous-time jump deterioration models", Journal of Quality in Maintenance Engineering, 12(3), pp. 306-329 (2006).

14. Iung, B. and Levrat, E. "Advanced maintenance services for promoting sustainability", Procedia CIRP, 22, pp. 15-22 (2014).

15. Jasiulewicz-Kaczmarek, M. "Sustainability: orientation in maintenance management: case study", EcoProduction and Logistics, pp. 135-154 (2013).

16. WCED, S.W.S. "World commission on environment and development", Our Common Future (1987).

17. Michaelis, L. "The role of business in sustainable consumption", Journal of Cleaner Production, 11(8), pp. 915-921 (2003).

18. Kalantary, M., Saen, R.F., and Eshlaghy, A.T. "Sustainability assessment of supply chains by inverse network dynamic data envelopment analysis", Scientia Iranica, Transactions E, Industrial Engineering, 25(6), pp. 3723-3743 (2018).

19. Fotuhi-Friuzabad, M., Safdarian, A., Moeini-Aghtaie, M., et al. "Upcoming challenges of future electric power systems: sustainability and resiliency", Scientia Iranica, 23(4), pp. 1565-1577 (2016).

20. Shahidehpour, M. and Fotuhi-Friuzabad, M. "Grid modernization for enhancing the resilience, reliability, economics, sustainability, and security of electricity grid in an uncertain environment", Scientia Iranica, Transaction D, Computer Science \& Engineering, Electrical, 23(6), p. 2862 (2016).

21. Ararsa, B.B. "Green maintenance: A literature survey on the role of maintenance for sustainable manufacturing", MS Thesis, Mälardalen University, School of Innovation, Design and Engineering (2012).

22. Kopac, J. "Achievements of sustainable manufacturing by machining", Journal of Achievements in Materials and Manufacturing Engineering, 34(2), pp. 180-187 (2009).

23. Ben-Daya, M., Ait-Kadi, D., Duffuaa, S.O., et al. Handbook of Maintenance Management and Engineering, 7, Springer, London (2009).

24. Sari, E., Shaharoun, A.M., Ma'aram, A., et al. "Sustainable maintenance performance measures: A pilot survey in Malaysian automotive companies", Procedia CIRP, 26, pp. 443-448 (2015).

25. Nezami, F.G. and Yildirim, M.B. "A sustainability approach for selecting maintenance strategy", International Journal of Sustainable Engineering, 6(4), pp. 332-343 (2013).

26. Mishra, R.P. and Mungi, P. "A system framework for a sustainable approach to maintenance", Sustainable Operations in India, pp. 79-91 (2018).
27. Li, S. "Optimal control of production-maintenance system with deteriorating items, emission tax and pollution R\&D investment", International Journal of Production Research, 52(6), pp. 1787-1807 (2014).

28. Ben-Salem, A., Gharbi, A., and Hajji, A. "Environmental issue in an alternative production-maintenance control for unreliable manufacturing system subject to degradation", The International Journal of Advanced Manufacturing Technology, 77(1-4), pp. 383398 (2015).

29. Hajej, Z., Rezg, N., and Gharbi, A. "Ecological optimization for forecasting production and maintenance problem based on carbon taxthem", The International Journal of Advanced Manufacturing Technology, 88(58), pp. 1595-1606 (2017a).

30. Ba, K., Dellagi, S., Rezg, N., et al. "Joint optimization of preventive maintenance and spare parts inventory for an optimal production plan with consideration of CO2 emission", Reliability Engineering \& System Safety, 149, pp. 172-186 (2016).

31. Hajej, Z., Rezg, N., and Gharbi, "A. Joint optimization of production and maintenance planning with an environmental impact study", The International Journal of Advanced Manufacturing Technology, 93(1-4), pp. 1269-1282 (2017b).

32. Franciosi, C., Lambiase, A., and Miranda, S. "Sustainable maintenance: A periodic preventive maintenance model with sustainable spare parts management", IFAC-Papers OnLine, 50(1), pp. 13692-13697 (2017).

33. Al-Turki, U.M., Ayar, T., Yilbas, B.S., and Sahin, A.Z., Integrated Maintenance Planning in Manufacturing Systems, Cham: Springer (2014).

34. Tlili, L., Radhoui, M., and Chelbi, A. "Conditionbased maintenance strategy for production systems generating environmental damage", Mathematical Problems in Engineering, 2015, Article ID 494162 (2015). https://doi.org/10.1155/2015/494162

35. Chouikhi, H., Khatab, A., and Rezg, N. "A conditionbased maintenance policy for a production system under excessive environmental degradation", Journal of Intelligent Manufacturing, 25(4), pp. 727-737 (2014).

36. Ben-Salem, A., Gharbi, A., and Hajji, A. "Production and uncertain green subcontracting control for an unreliable manufacturing system facing emissions", The International Journal of Advanced Manufacturing Technology, 83(9-12), pp. 1787-1799 (2016).

37. Jiang, A., Dong, N., Tam, K.L., et al. "Development and optimization of a condition-based maintenance policy with sustainability requirements for production system", Mathematical Problems in Engineering, 2018, Article ID 4187575 (2018). https://doi.org/10.1155/2018/4187575

38. Alaswad, S. and Xiang, Y. "A review on conditionbased maintenance optimization models for stochastically deteriorating system", Reliability Engineering \& System Safety, 157, pp. 54-63 (2017). 
39. Papakonstantinou, K.G. and Shinozuka, M. "Planning structural inspection and maintenance policies via dynamic programming and Markov processes. Part I: theory", Reliability Engineering \& System Safety, 130, pp. 202-213 (2014).

40. Kumar, A. and Meenakshi, N., Marketing Management, Vikas Publishing House (2011).

41. Ahmadi-Javid, A. and Ghandali, R. "An efficient optimization procedure for designing a capacitated distribution network with price-sensitive demand", Optimization and Engineering, 15(3), pp. 801-817 (2014).

42. Pak, P.K., Kim, D.W., and Jeong, B.H. "Machine maintenance policy using partially observable Markov decision process", Journal of the KSQC, 16(2), pp. 1-9 (1988).

43. Kaelbling, L.P., Littman, M.L., and Cassandra, A.R. "Planning and acting in partially observable stochastic domains", Artificial Intelligence, 101(1), pp. 99-134 (1998).

44. Pineau, J., Gordon, G., and Thrun, S. "Point-based value iteration: An anytime algorithm for POMDPs", IJCAI, 3, pp. 1025-1032 (2003).

45. Spaan, M.T. and Vlassis, N. "Perseus: Randomized point-based value iteration for POMDPs", Journal of Artificial Intelligence Research, 24, pp. 195-220 (2005).

46. Raphael, C. and Shani, G. "The skyline algorithm for POMDP value function pruning", Annals of Mathematics and Artificial Intelligence, 65(1), pp. 61-77 (2012).

47. Karmokar, A.K., Senthuran, S., and Anpalagan, A. "POMDP-based cross-layer power adaptation techniques in cognitive radio networks", Global Communications Conference, IEEE, pp. 1380-1385 (2012).

48. Roijers, D.M., Vamplew, P., Whiteson, S., et al. "A survey of multi-objective sequential decision-making", Journal of Artificial Intelligence Research, 48, pp. 67113 (2013).

49. Li, D. and Jayaweera, S.K. "Machine-learning aided optimal customer decisions for an interactive smart grid", IEEE Systems Journal, 9(4), pp. 1529-1540 (2015).

50. Qian, W., Liu, Q., Zhang, Z., et al. "Policy graph pruning and optimization in Monte Carlo value iteration for continuous-state POMDPs", In Computational Intelligence (SSCI), Symposium Series on IEEE, pp. 1-8 (2016).

51. Walraven, E. and Spaan, M.T. "Accelerated Vector Pruning for Optimal POMDP Solvers", In AAAI, pp. 3672-3678 (2017).

52. Bellman, R., Dynamic Programming, Courier Corporation (2013).

53. Ahuja. D.https://www.codeproject.com/Articles/9898 /Heap-Walker (2005).
54. Agrawal, R., Realff, M.J., and Lee, J.H. "MILP based value backups in partially observed Markov decision processes (POMDPs) with very large or continuous action and observation spaces", Computers \& Chemical Engineering, 56, pp. 101-113 (2013).

55. Smallwood, R.D. and Sondik, E.J. "The optimal control of partially observable Markov processes over a finite horizon", Operations Research, 21(5), pp. 10711088 (1973).

56. Özgen, S. and Demirekler, M. "A fast elimination method for pruning in POMDPs", Joint German/Austrian Conference on Artificial Intelligence, Springer International Publishing, pp. 56-68 (2016).

57. Feng, Z. and Zilberstein, S. "Region-based incremental pruning for POMDPs", 20th Conf. on Uncertainty in Artificial Intelligence, AUAI Press, pp. 146-153 (2004).

58. Cassandra, A., Littman, M.L., and Zhang, N.L. "Incremental pruning: A simple, fast, exact method for partially observable Markov decision processes", 13th Conf. on Uncertainty in Artificial Intelligence, Morgan Kaufmann Publishers Inc., pp. 54-61 (1997).

59. White, C.C. "A survey of solution techniques for the partially observed Markov decision process", Annals of Operations Research, 32(1), pp. 215-230 (1991).

60. Littman, M.L., The Witness Algorithm: Solving Partially Observable Markov Decision Processes, Brown University, Providence, RI. (1994).

\section{Biographies}

Razieh Ghandali is currently a PhD Candidate of Industrial Engineering at Yazd University, Yazd, Iran. She obtained an MS degree in Industrial Engineering from Amir Kabir University of Technology (Tehran, Iran) in 2011 and a BS degree in Industrial Engineering from Alzahra University (Tehran, Iran) in 2006. Her research interests include operations research, supply chain, time series, dynamic programming, uncertainty modeling, and Markov models.

Mohammad Hossein Abooie is currently an Assistant Professor of Industrial Engineering at Yazd University. He received his BS, MS, and PhD degrees in Industrial Engineering from Amir Kabir University of Technology in 1993, 1995, and 2009, respectively. His research interests include quality control and management, customer relationship management and marketing, economics, and applied operations research.

Mohammad Saber Fallah Nezhad is currently an Associate Professor of Industrial Engineering at Yazd University. He received his $\mathrm{BS}, \mathrm{MS}$, and $\mathrm{PhD}$ degrees in Industrial Engineering from Sharif University of Technology in 2003, 2005, and 2008, respectively. His research interests include quality control, Bayesian inference, dynamic programming, and Markov models. 